\title{
Recipient design in tacit communication
}

\author{
Sarah E. Newman-Norlund ${ }^{\mathrm{a}, *}$, Matthijs L. Noordzij ${ }^{\mathrm{a}, \mathrm{d}}$, Roger D. Newman-Norlund ${ }^{\mathrm{a}, \mathrm{b}}$, \\ Inge A.C. Volman ${ }^{\mathrm{a}}$, Jan Peter de Ruiter ${ }^{\mathrm{c}}$, Peter Hagoort ${ }^{\mathrm{a}, \mathrm{c}}$, Ivan Toni ${ }^{\mathrm{a}, \mathrm{b}}$ \\ ${ }^{a}$ Donders Institute for Brain, Cognition and Behaviour, Centre for Cognitive Neuroimaging, Radboud University Nijmegen, The Netherlands \\ ${ }^{\mathrm{b}}$ Donders Institute for Brain, Cognition and Behaviour, Centre for Cognition, Radboud University Nijmegen, Nijmegen, The Netherlands \\ ${ }^{\mathrm{c}}$ Max Planck Institute for Psycholinguistics, Nijmegen, The Netherlands \\ ${ }^{\mathrm{d}}$ Department of Cognitive Psychology and Ergonomics, University of Twente, The Netherlands
}

\section{A R T I C L E I N F O}

\section{Article history:}

Received 4 February 2008

Revised 11 December 2008

Accepted 19 December 2008

\section{Keywords:}

Audience design

Motherese

Nonverbal

Social game

Empathy

\begin{abstract}
A B S T R A C T
The ability to design tailored messages for specific listeners is an important aspect of human communication. The present study investigates whether a mere belief about an addressee's identity influences the generation and production of a communicative message in a novel, non-verbal communication task. Participants were made to believe they were playing a game with a child or an adult partner, while a confederate acted as both child and adult partners with matched performance and response times. The participants' belief influenced their behavior, spending longer when interacting with the presumed child addressee, but only during communicative portions of the game, i.e. using time as a tool to place emphasis on target information. This communicative adaptation attenuated with experience, and it was related to personality traits, namely Empathy and Need for Cognition measures. Overall, these findings indicate that novel nonverbal communicative interactions are selected according to a socio-centric perspective, and they are strongly influenced by participants' traits.
\end{abstract}

(c) 2008 Elsevier B.V. All rights reserved.

\section{Introduction}

Recipient design is the adaptation of communicative behavior to a particular addressee (Garfinkel, 1967). This is one instance of audience design (Clark \& Carlson, 1982), in which utterances are constructed according to what others in the communicative exchange know and believe (Clark, 1996). Audience design has been reported in speech and actions in both naturalistic and experimental settings (Holler \& Stevens, 2007; Jacobs \& Garnham, 2007; O’Neill, Bard, Linnell, \& Fluck, 2005; Ozyurek, 2002), but there is still considerable debate over when, how, and why it is used. An outstanding issue is whether audience design is structurally linked to initial attempts to communicate, or whether it is used

\footnotetext{
* Corresponding author. Tel.: +31 024366 6275; fax: +31 0243612620 E-mail address: serieliz@gmail.com (S.E. Newman-Norlund).
}

parsimoniously to repair erroneous attempts. Some authors support a socio-centric approach, in which communicative behavior is designed by taking into account the knowledge and characteristics of the addressee that are assumed to be part of common ground (Clark, 1996; Isaacs \& Clark, 1987; Nadig \& Sedivy, 2002). Other authors suggest that the initial planning of utterances ignores common ground (egocentric approach) and that messages are adapted to addressees only when adjustments are required (Horton \& Keysar, 1996; Keysar, Barr, \& Horton, 1998).

In the current experiment, we test whether communicators adapt their messages to an important component of common ground information, i.e. their conceptual model of the addressee, while controlling for factors that might account for some of the conflicting empirical evidence (Barr, 2004; Brown-Schmidt, Gunlogson, \& Tanenhaus, 2008; Keysar et al., 1998). For instance, cognitive load of the communicative task, accessibility to memory representation, 
and availability of communicative feedback may bias participants to adopt either a socio-centric or an ego-centric approach (Horton \& Gerrig, 2005). Furthermore, given our "tentative and probabilistic" knowledge of what others know (Krauss \& Fussell, 1991), variability in individual cognitive abilities might influence the use of audience design. For instance, it has been found that individuals high in systemizing abilities are superior at tasks such as map-reading and mathematics, while people with high empathizing abilities are better at predicting the actions of others and responding to these in appropriate ways (Saxe \& BaronCohen, 2006). Accordingly, individuals with high empathizing scores (here measured by the Empathizing Quotient (EQ), (Baron-Cohen \& Wheelwright, 2004) may be more likely to adapt communicative message, since they can better predict the response of others, while those with high systemizing abilities (SQ-R) may take a more logical problem-solving approach to communicative efforts due to their increased tendency to search for rules and definitive answers. Individuals high in Need for Cognition (NCS) are flexible in choosing learning strategies, are highly motivated, and have good control over attentional resources, while those low in need for cognition have the tendency to rely on others to find meaning in events and stimuli (Cacioppo, Petty, \& Kao, 1984; Evans, Kirby, \& Fabrigar, 2003).Similarly, individuals high in Need for Cognition (Cacioppo, Petty, Feinstein, Blain, \& Jarvis, 1996) are less influenced by surface information (Ruiter, Verplanken, De Kremer, \& Kok, 2004) and could be less likely to adapt their behavior to the observable characteristics of a communicative partner.

In this study, we exploit the naturally occurring communicative adaptations observed when adult communicators direct their speech, gestures, and accompanying motions towards child addressees (Brand, Baldwin, \& Ashburn, 2002; Brodsky, Waterfall, \& Edelman, 2007; Grieser \& Kuhl, 1988; O’Neill et al., 2005; Warren-Leubecker \& Bohannon, 1984). To this end, we test whether the mere belief that one is communicating with either a child or an adult addressee is sufficient to induce communicative adaptations in adults. We elaborated on the ideas of the Tacit Communication Game or TCG (De Ruiter, Noordzij, Newman-Norlund, Hagoort \& Toni, 2007). In the current game, the players need to communicate the spatial location of a target to their partner using both simple and complex messages. On communicatively simple trials, players can easily and unambiguously express the location of the target by moving to the single target location, while on difficult trials players need to generate a novel communicative action to express the location of the target amongst multiple potential locations. This task made it possible to control production and effectiveness of communications and exactly match the behavior of child and adult addressees, while preserving relevant aspects of human communication (i.e. joint goal, collaboratively established common ground, information sharing). We assess whether adults adapt their communicative behavior towards their presumed partners, whether these adaptations are confined to communicative actions, whether the extent of adaptation varies with communicative complexity, and whether the magnitude of adaptation is related to personality traits.
Paralleling findings with infants and children (Brodsky et al., 2007; Warren-Leubecker \& Bohannon, 1984), we predict adaptations will be found in the rate of communication (movement time), the emphasis placed on key elements (time on target location), and in the repetition of important information (number of moves). We also explore effects of recipient traits on planning time and the effect of communicative complexity.

\section{Methods}

\subsection{Participants}

Twenty-four right-handed females (ages 18-30 years) took part in this study. All participants gave informed consent according to institutional guidelines of the local ethics committee (CMO Arnhem-Nijmegen, The Netherlands), and were either offered financial payment or credits towards a course requirement. The purpose of the experiment was explained to all participants upon completion. Two participants did not believe they were playing with a real child or adult and were excluded from the study. One participant did not complete the personality measurements and was excluded from the individual differences analyses.

\subsection{Design and materials}

The game involves a Sender (portrayed as a blue bird) and a Receiver (portrayed as a squirrel), moving on a $3 \times 3$ grid designed to look like a park with 9 square areas (Fig. 1a). Each Sender played the game on a 19 in. computer monitor using a Logitech hand-held controller. The spatial lay-out of the buttons on the controller allowed for mappings between finger and token movements: four face buttons moved the token to the left, right, up and down; a left-shoulder button was used as a start/end button. The blue bird token could only move one square at a time to the left, right, up, or down, so it was not possible for the bird to move diagonally in one move. Furthermore, the bird could only move to the center of each of the 9 squares in the park. The confederate receiver moved with a mouse that showed continuous on-screen movement until they clicked the left mouse button. The Sender sat facing the monitor in a sound-proof experimental room. The game was programmed using Presentation software (version 9.90 Neurobehavioral Systems, Davis, CA) run on a Dell Workstation (Austin, TX, USA).

\subsection{Procedure}

Each participant completed a familiarization session (10 trials) ensuring that participants understood the task and learned the mapping between the controller buttons and on-screen movements. The Sender was instructed to move her playing token (a blue bird) to the location of a target token (berries), as indicated during the presentation of the goal configuration (Fig. 1a). When the Sender pressed the start button on the hand-held controller, the berries disappeared, the blue bird moved to the center of the 
A

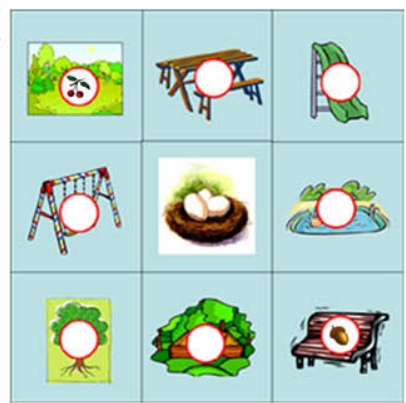

1.

B

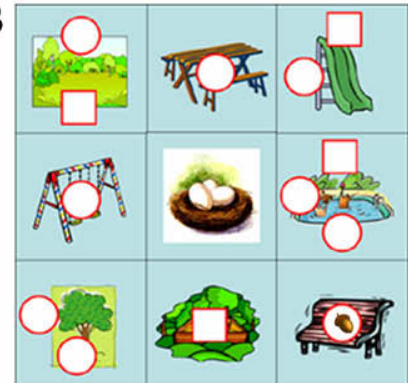

1.

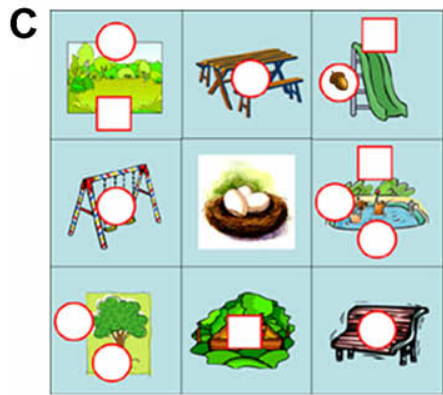

1.

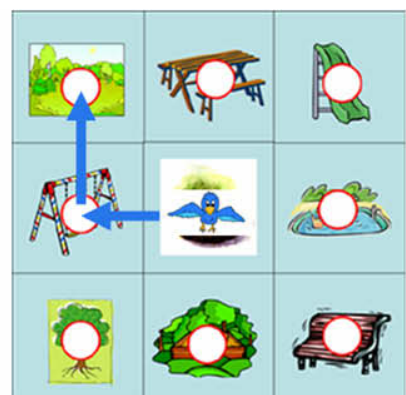

2.

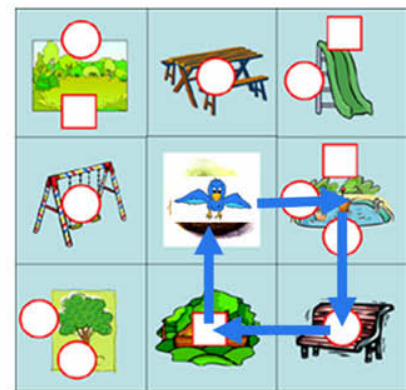

2.

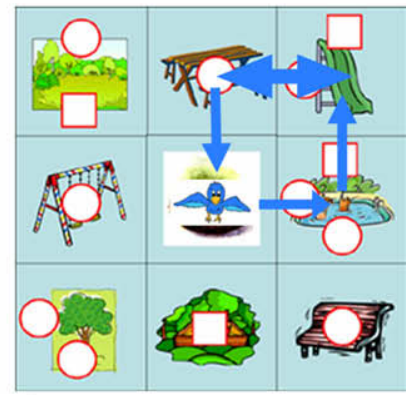

2.

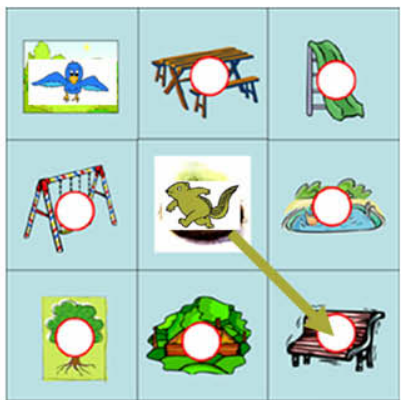

3.

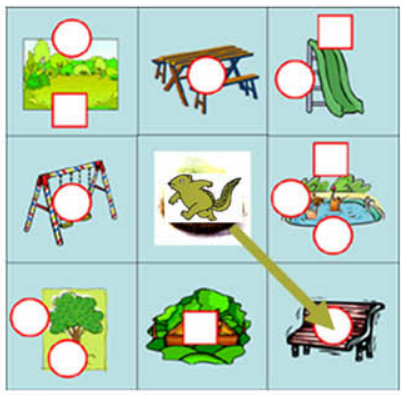

3.

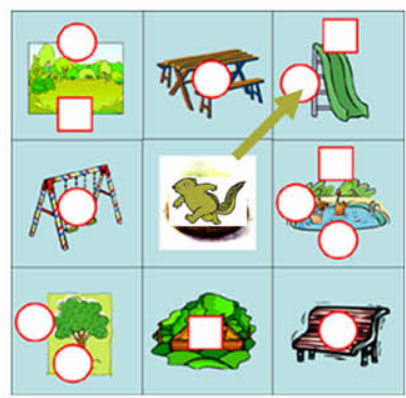

3.

Fig. 1. Task design. Examples of gameboards in different trial types, as seen by the Sender. Panels in columns 1,2 , and 3 indicate successive stages of the game during a single trial, namely 1) the initial board configuration (visible to both Sender and Receiver during Control trials, and ONLY to the Sender during Communicative trials), 2) the movements of the Sender (represented by the bird), and 3) the movements of the Receiver (the squirrel) towards the seen (Control trials) or inferred (Communicative trials) location of the acorn. Panels in row A, B, and C illustrate Control, Communicative Easy, and Communicative Difficult trials, respectively. Arrows are used to illustrate movements on the gameboard (bird's and squirrel's movements are indicated by blue and brown arrows; no arrows were displayed during the game). Note that the bird could only move to the center of each of the nine square areas. A.) Control Trials: Each of the nine square areas contains a single potential target location (white circle). The Sender was asked to move her game token (a bird) to the target location containing the berries. The Receiver was asked to move her game token (a squirrel) to the target location containing the acorn. Communicative Trials: The target (acorn) was only visible to the Sender. The Sender's task was to communicate where the acorn could be found. B.) Easy Communicative Trials: The square area where the acorn was located contains only one target location (white circle or square). Movement to the square area containing the acorn unambiguously indicated where the acorn is. C.) Difficult Communicative Trials: The square area where the acorn was located contains more than one white circle/square, driving the Sender to find novel communicative actions to indicate which of the 2 or 3 locations was correct. For instance, the Sender wiggled back and forth across the border of two target squares (wiggling is indicated by the double headed arrow).

gameboard, and the Sender had a time limit of ten seconds to move the blue bird to the berry location with the handheld controller. This limit on movement time was used to maximize the potential to isolate the separate processes of planning and execution of the Sender's movements while allowing adequate time to respond. After $10 \mathrm{~s}$ or when the Sender pressed the 'done' button, visual feedback (500 ms) indicated whether the movement was correct (green square) or incorrect (red square).
Each Sender was previously instructed that they would be playing an interactive computer game with two partners (Receivers; a child and adult), in alternation and that they would see a photograph of their current partner before and during each session. An experimental confederate entered the room and stated that the other two partners were ready. A digital photograph of the Sender was taken, and after a short delay the experiment began. There were two types of trials, control and Communicative trials. 
During CONTROL trials, both players could see the target locations (Fig. 1.A). The Sender was instructed to move her bird token to the target location (red berries). The Receiver was required to move her playing token (squirrel) to the location of her target (acorn). The location of the two targets was indicated during the presentation of the goal configuration at the beginning of each trial (Fig. 1.A1). When the Sender pressed the start button, the berries and the acorn disappeared from the gameboard, the bird appeared in the center square (Fig. 1.A2), and the Sender had ten seconds to move the bird to the location of the berries. Sender's controller movements had limited degrees of freedom, only allowing movements to the center of each of the nine squares in the park. Upon the Sender pushing the end button or time elapsing, the Receiver moved the squirrel to the location of the acorn using a mouse with unlimited degrees of freedom (Fig. 1.A3). Correct feedback was conditional on both players moving to the location of their respective target tokens within the allotted time.

During Communicative trials, the location of the acorn was only shown to the Sender (Fig. 1 B1 \& C1). In order to successfully complete the trial, the Sender needed to communicate to the Receiver the location of the acorn. Apart from this difference, COMMUNICATIVE trials followed the same time course of events as CONTROL trials. Successful trials, in which the Receiver moved to the location of her target token and the Sender returned to the bird's nest within the allowed time, resulted in the presentation of an acorn on the screen. A red "no" icon was presented over the acorn for unsuccessful trials. We further distinguished between easy and difficult COMMUNICATIVE trials, depending on whether the acorn was positioned in a square of the park with one (easy) or more (difficult) potential target location(s) - (Fig. 1 B versus C). Due to limitations on the Sender's movements, to the middle of each square on the gameboard, difficult trials required the generation of novel communicative actions to express which of the 2 or 3 locations in the square was the correct one. The Sender's actions in easy trials typically entailed moving to the location of the acorn, pausing, and returning to the nest (See Fig. 1-B2). For difficult trials, Senders could communicate the location of the acorn by using different strategies, for instance using the "route" to the target square (approaching the square from above if the target location was at the top of the square); or wiggling back and forth across the border of two squares (if the target location was close to this border) (See Fig. 1-C2).

Trials were organized by Trial type and Receiver type into 8 blocks (Fig. 2). Easy and difficult trials were intermixed in Communicative blocks and equally distributed across the 4 sessions of communicative trials.

Unbeknownst to the Sender, there was only one confederate Receiver during the test sessions (S.E.N.N.). The Receiver did not know whether the Sender was made to believe she was playing with a Child or Adult Receiver. Performance of the confederate Receiver was pre-set for both Adult and Child Receivers: 95\% for Control, 96\% for Easy, and $52 \%$ for difficult trials. This pre-set performance was achieved by placing a marker ("go here") on the location to which the confederate receiver should move her mouse. The Receiver had the option of deviating from the auto- matically suggested response location if the Sender did not comply with task instructions or made an error in their performance. For example, the Sender might press the start button before they saw where the target(s) was located, and then signal the wrong (any or no) target location. If the Receiver noted a discrepancy between the Sender's movements and the Receiver's target square, the Receiver would choose a more appropriate response (no indication of location= staying on the nest; wrong target location=choosing the wrong target location). This ensured that the Sender believed they were playing with a real child and adult and not a computer simulation with automatic responses. Accordingly, there were small variations from the pre-set performance (see manipulation checks).

\subsection{Questionnaires/scales}

Participants completed a questionnaire after the experimental session that addressed characteristics about their partners and general questions pertaining to the game and the strategies they used (see Appendix A). This provided an opportunity for participants to indicate that they did not believe the receiver manipulation. Participants completed three personality scales, the Dutch versions of the EQ (Baron-Cohen \& Wheelwright, 2004), the SQ-R (Wheelwright et al., 2006), and the NCS (Cacioppo et al., 1996).

\subsection{Data analysis}

Independent variables were all varied within participants and included Receiver_Type (Child or Adult) and Trial_Type (Control, Easy_Communicative or Difficult_Communicative). Dependent measures of movement time, and time spent on target location, planning time, and number of moves were recorded by Presentation 9.90 software. Movement time refers to the time from when the Sender pushed the start button (to begin their movement) until the last button press of that trial, which was typically the "end" button. If the Sender did not press the "end" button, the last movement button pressed was used for this measure. Time spent on the target location was calculated for each trial as the difference between the occurrence of the button press that moved the blue bird token into the target location and the occurrence of the subsequent button press that moved the token into any other location. When the token hit the target location multiple times within a trial, the mean of these differences was considered. Likewise, the time spent on non-target locations was calculated as the mean of the differences between the occurrence of the button presses that moved the blue bird token into a specific non-target location, and the occurrence of the button presses that moved the token into any other location (target, middle square, or another nontarget). Planning time was the time from when the target(s) appeared on the screen until the Sender pressed the start button to begin moving the bird. Number of moves refers to the number of button presses that produced a visible movement on the screen.

Mean movement time, time spent on target location, planning time, and number of moves for the Sender were 
Experimental timeline

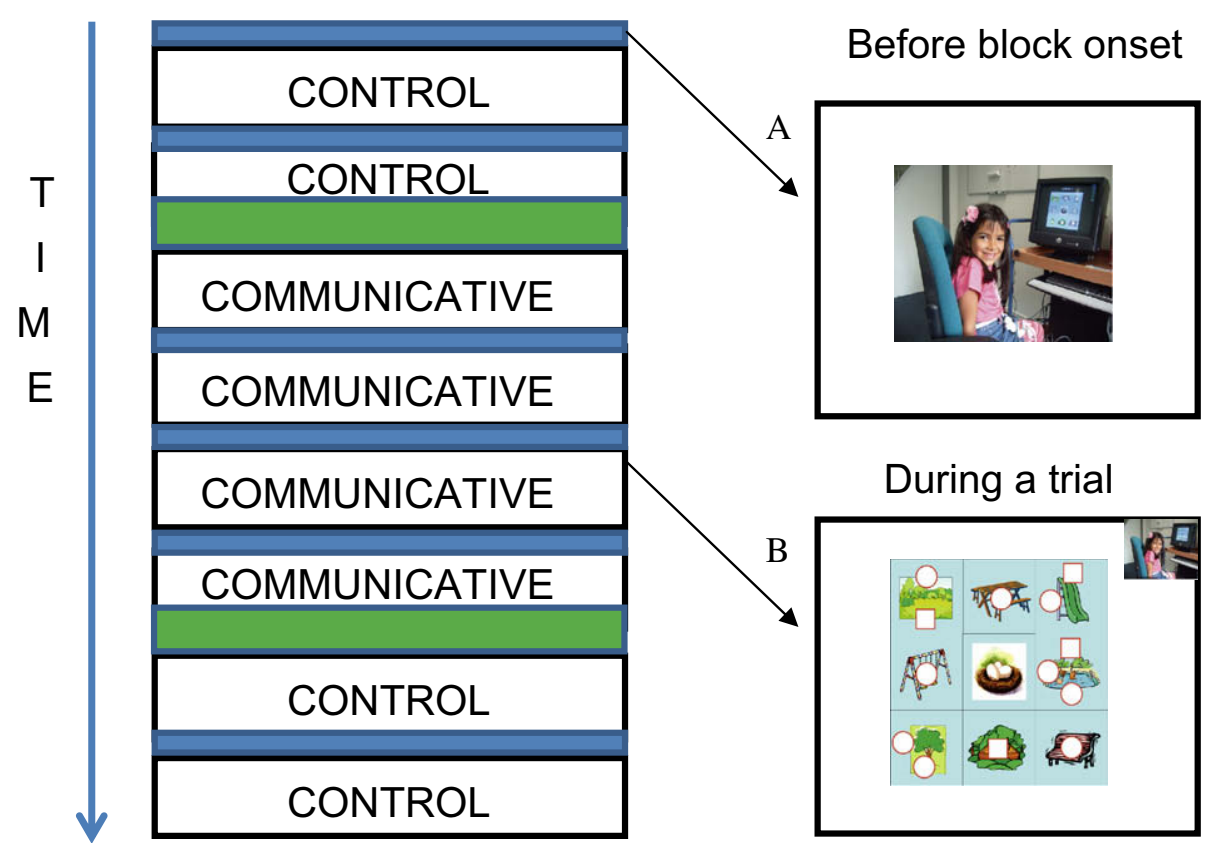

Fig. 2. Timeline of Experimental Protocol and Examples of Screen Presentation. Participants started the experimental session by playing a block of 10 control trials with each of the two receiver types (adult, child), and then continued playing four blocks of communicative trials (24 trials each), concluding with two blocks of control trials ( 10 trials each. Before each block of trials started, a picture of the forthcoming Receiver was displayed in the middle of the screen (panel A) for one minute (thinner blue boxes). During each trial, a picture of the current Receiver was displayed in the upper right hand corner of the screen (panel B). Receiver type alternated between successive blocks, with order counterbalanced across subjects. At the transition between control and communicative trials, the picture was shown for 3 min (thicker green boxes).

analyzed using separate $2 \times 3$ repeated measures ANOVAs. Huynh-Feldt correction was applied in all tests involving the variable Trial Type to correct for possible violations of sphericity.

\section{Results}

\subsection{Movement time}

The main effects of Receiver_Type and Trial_Type on movement time were significant, $F(1,21)=8.4, p=0.009$ and $F(2,42)=58.6, p<0.001, \varepsilon=0.6$. Participants took more time to move when they thought the Receiver was a child. Movement times were also longer for Difficult than Easy trials, $t(21)=6.5, p<0.001$, and for Easy than Control trials, $t(21)=7.7, p<0.001$. Importantly, the interaction was also significant, $F(2,42)=3.6, p=0.047, \varepsilon=0.8$, indicating that participants had longer movement times for the child Receiver compared to the adult Receiver but only in the Communicative trials (Easy trials, $t(21)=3.5, p=0.002$, Difficult trials, $t(21)=2.1, p=0.045)$, and not in the Control trials $(t(21)<1)$ (Fig. 3).

As a follow-up, we investigated how this additional movement time in Communicative trials was spent for Child Receivers, assessing whether it was driven by additional emphasis (longer pauses) on the target location. Accordingly, we contrasted time spent on target location versus non-target locations for Adult and Child Receivers using a $2 \times 2$ ANOVA. The main effect of target/non-target location was significant, $F(1,21)=23.7, p<0.001$, indicating that participants kept their token much longer on the target position than on a non-target position. As before, the main effect of Receiver_Type was significant, $F(1,21)=7.2, p=0.01$, showing that time spent on target

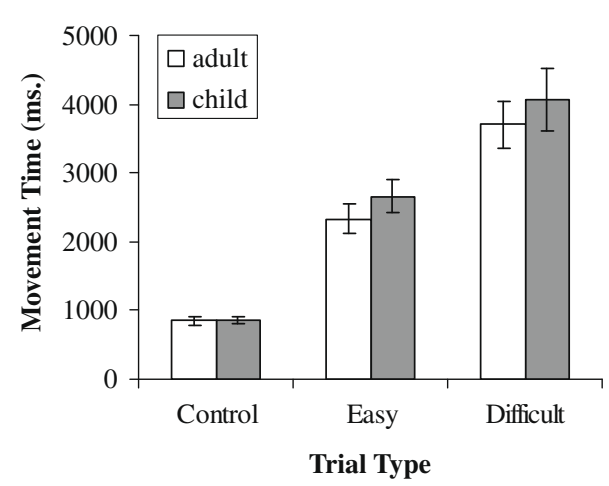

Fig. 3. Movement times (mean \pm SEM) of the Sender, directed towards a presumed adult or child receiver, during different trial types. It can be seen that participants slowed down their movements when directed to child Receiver (compared to the adult Receiver) only in the Communicative trials and not in the Control trials. 


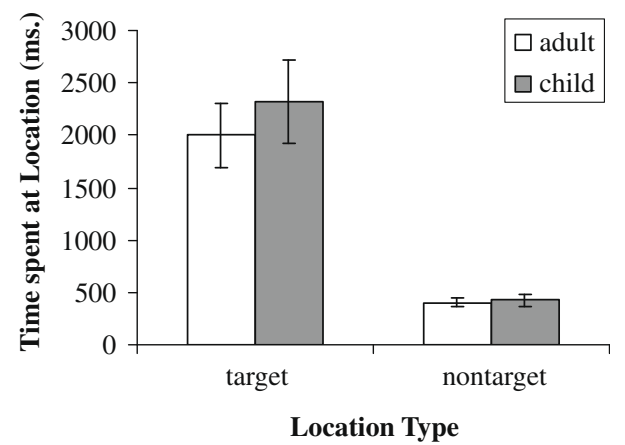

Fig. 4. Time (mean \pm SEM) of the Sender spent on target and non-target locations as a function of the presumed partner (adult/child receiver). It can be seen that participants spent more time at the target locations for child than adult partners.

was longer for a Child receiver. The interaction between target/non-target location and Receiver_Type was also significant, $F(1,21)=4.8, p=0.04$ (Fig. 4). Players spent significantly more time at the target locations for child than adult partners $(t(21)=2.4, p=0.02)$. This was not the case for non-target locations $(t(21)=1.4 ; p=0.18)$.

Furthermore, we evaluated whether there was an adaptation in behavior based on performance monitoring. COMMUNICATIVE blocks were split in half (first and last 12 trials of a block). A repeated measures ANOVA $(2 \times$ $2 \times 2$ ) was run on Receiver_Type (Child, Adult) over Block (First Half, Second Half) for EASY and DIFFICULT trials. The interaction of Receiver_Type by Block was significant $(F(1,21)=4.75 ; p=0.041)$. Movement times decreased over the course of a block for child partners $(t(21)=2.56$; $p=0.018$ ), while there was no significant change for adult partners $(\mathrm{t}(21)=-0.144 ; p=0.89 ;$ Fig. 5). All other interactions were not significant (Fs $<1.1$; ps $>0.30$ ). An equivalent analysis based on the whole block revealed the same qualitative pattern, but failed to reach statistical significance.

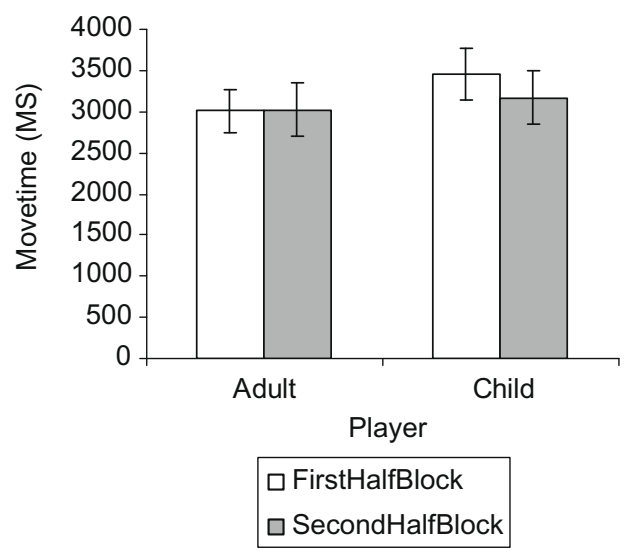

Fig. 5. Movement time (mean \pm SEM) of the Sender during the first and the second half of the game sessions as a function of the presumed partner (adult/child receiver).

\subsection{Planning time and number of moves}

There was a main effect of Trial_Type for both the planning time and number of moves of participants $(F(2,42)=$ 28.3, $p<0.001, \varepsilon=0.57 ; \quad F(2,42)=109.9, p<0.001, \varepsilon=$ 0.58). Participants had longer planning times $(t(21)=5.3$, $p<0.001 ; t(21)=2.9, p=0.009)$ and made more moves $(t(21)=7.3, p<0.001 ; t(21)=16.5, p<0.001)$ for Difficult versus Easy trials, and Easy versus Control trials. All other effects were not significant ( $F s<2$; ps $>0.17$ ).

\subsection{Individual differences}

We evaluated whether EQ, SQ-R, and NCS could explain the degree of difference in player's movement times associated with child and adult Receivers in COMMUNICATIVE trials. A stepwise linear regression was performed with EQ, SQ-R, and NCS scores on the difference between movement times for adult and child partners. Difference scores were calculated for each participant (mean Child Movetimemean Adult Movetime). NCS and EQ were statistically significant predictors of the movement difference scores (NCS: $B=-0.517, p=0.026 ; \quad E Q: \quad B=0.492, p=-014$ ) (Fig. 6); while SQ-R was not a significant predictor $(t<1)$. Combined model of EQ and NCS account for substantial variability in adaptation, adjusted $R^{2}=.403$. Individuals with high NCS were less likely to have longer movetimes for the child as compared with the adult partner. Individuals with high EQ scores showed the greatest differences in Movetime between child and adult partners. Individual with high NCS are explorative in their thinking, and good at spatial and reasoning tasks. Perhaps in this task, participants focus more on finding a logically optimal solution to the task, rather than considering how to adapt their message to their partner. On the other hand, individuals high in empathy are typically better at reading and adapting to the intentions of others, and therefore were more likely to use this personal information as a tool in communicating. Taken together, it appears that individuals vary in how much and whether they will adapt their communicative messages (in this case to a child or adult addressee) based on cognitive traits of empathy and affinity for complex thought.

While SQ-R was not a valid predictor in the regression model, we further explored this variable and found that the SQ-R and NCS scores are highly correlated $(R=.562$; $p=.008)$. When taken alone, SQ-R is related to the differential adaptation in movetime, $(\mathrm{t}(1,21)=-1.9 ; p=.07)$, but it is not as good of a predictor as NCS.

\subsection{Manipulation checks}

Participants were asked to guess the age of their child and adult partners. The mean $( \pm S D)$ age of each receiver reported by the participants was $8.1 \pm 4.2$ years (Child) and $25.6 \pm 1.2$ years (Adult). 11 participants explicitly reported in the follow-up questionnaire that they took more time to communicate with the child than with the adult partner.

There were no statistical differences in the performance of the confederate across Receiver_Type, either in terms of 


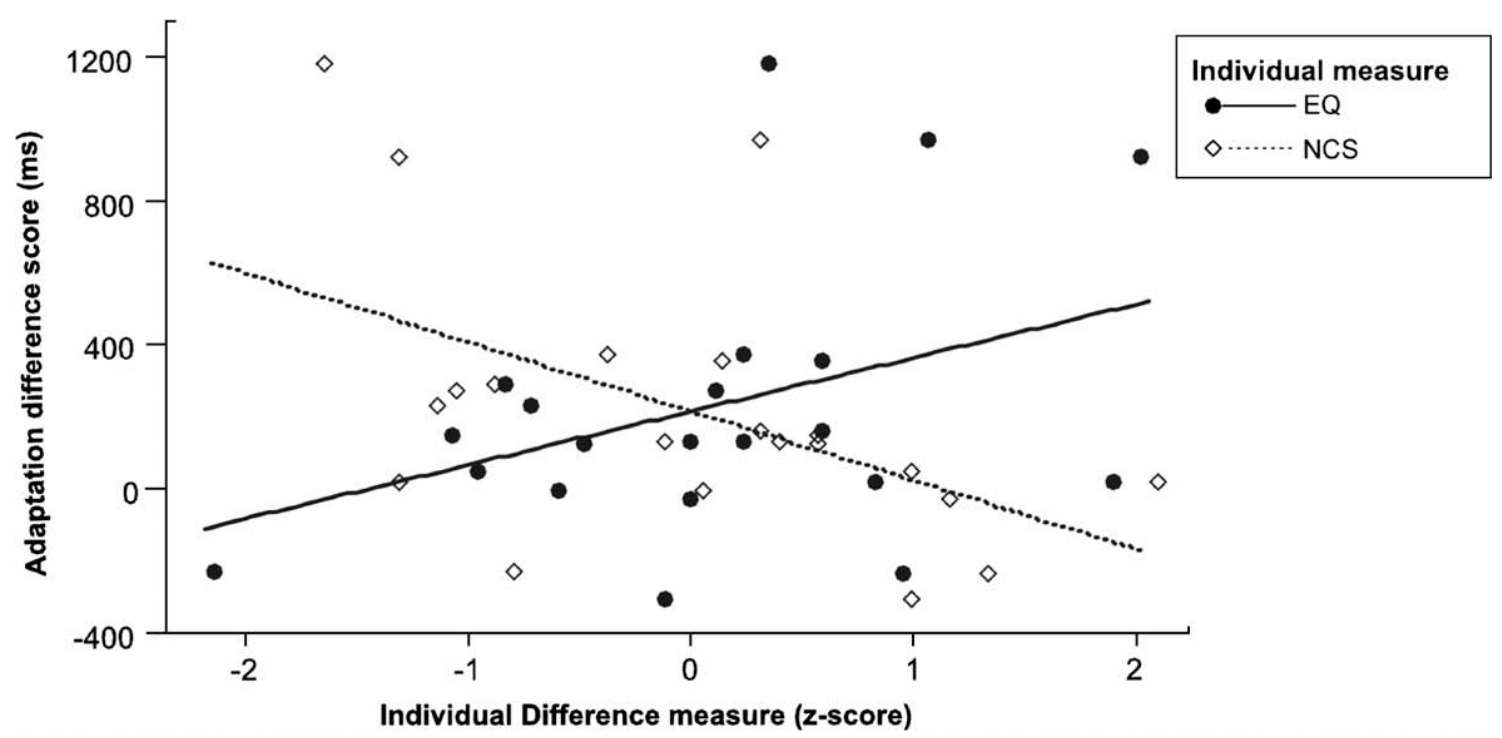

Fig. 6. Individual adaptation difference scores (ms) plotted against the z-scores of the EQ (black circles) and NCS (open diamonds) scores. Regression lines are plotted separately for EQ (solid line) and NCS scores (dotted line).

correct rates (Difficult trials: Child $=51.6 \%$, Adult $=51.8 \%$; Easy trials: Child $=95.2 \%$, Adult $=94.8 \%$ ) or in terms of response times (effect of Receiver_Type: $\mathrm{F}(1,42)=2.575$. $p$ $=0.12$; Receiver_Type $\mathrm{X}$ Trial_Type: $\mathrm{F}(2,42)=0.58$; $p=0.52$ ).

There was a difference in the confederate Response times as a function of Trial_Type $(\mathrm{F}(2,42)=87.424 ; p<$ 0.001 ), i.e. with response time being shortest for control trials and easy communicative trials, and longer for difficult communicative trials.

Confederate response times were also evaluated over block (first half block, second half block) as tested with the Sender's movement times. For the interaction Receiver_Type by Block, there were no significant differences, $(F(1,21)=2.03, p=0.17)$. The main effect of half block was significant $(F(1,21)=109.28, p<0.001)$, receivers responded faster in the second half of each block than in the first half for both receivers, child and adult. The main effect of Receiver_type was not significant, $(F(1,21)=$ $1.95, \mathrm{p}=0.18$ ).

We also conducted an analysis to assess the effect of Order of Receiver_type on Movement times. All analyses were not significant (All F's $<2.3$; p's > 0.14).

\section{Discussion}

We used a nonverbal communication task to test whether the behavior of communicators could be altered by the mere belief of addressing a child or adult, other factors being equal. We show that, in an environment with no pre-existing communicative conventions, participants adapt their communicative behavior to the presumed cognitive abilities of the addressee, putting greater emphasis on crucial communicative elements for child recipients. Below, we elaborate on the implications of these findings for current models of audience design in human communication.

While a confederate blindly acted as both child and adult partners with matched performance and response times, participants estimated the two presumed addressees to be 8 and 26 years old, respectively. Crucially, that belief influenced their communicative, but not their functional behavior (Fig. 3). Participants spent more time communicating with the presumed child than with the adult addressee, pausing distinctly longer at the target position in the former case (Fig. 4). This effect was not associated with equivalent functional motoric actions or with the planning complexity of those actions. Utilizing timing as a diacritic to mark communicatively relevant information closely matches similar adaptations found in adult-infant and adult-child interactions (i.e. use of prosodic emphasis of important words and use of higher pitch to draw and maintain attention to key aspects (Brodsky et al., 2007; Warren-Leubecker \& Bohannon, 1984).

While communicative complexity did result in longer movement times, planning times, and increased number of moves, it did not have a differential impact based on the presumed partner. In both easy and difficult communicative trials, participants exacerbated the use of timing to place more emphasis on the target location for the child partner. We did not find overall differences in the strategies that participants used, as seen in their number of moves. Participants also reported not wanting to confuse their partners by changing strategies. These findings are in line with the notion that "speakers" try to be consistent in their communicative manners (Shintel \& Keysar, 2007).

The communicative adaptation observed in this study was not a contagion/priming effect (Bargh, Chen, \& Bur- 
rows, 1996). The adaptation was absent during noncommunicative trials and during movements over nontarget locations and was voluntarily reported by half of the participants. Furthermore, as evidence accumulated regarding the addressees' performance, the magnitude of the communicative adaptation to the child addressee decreased, converging towards adult-directed communicative actions (Fig. 5). In other words, participants began each block making adaptations based on conceptual traits of the child and adult partners and during each block adapted their behavior to their current experience (performance). This corresponds with the findings of Horton and Gerrig (2005), whereby greater access to memory representations leads to greater adaptation of the speaker's behavior. Access to memory and information regarding performance increased over the block, making conceptual traits less salient. This finding introduces a dynamic perspective into the gricean maxim of Quantity (Grice 1969), i.e. participants made their contributions as informative as required, learning to avoid making them more informative than required.

Finally, participants with different personality traits showed different degrees of communicative adaptation to the child addressee: greater empathy (EQ) evoked larger adaptation, higher affinity for complex thought (NCS) evoked smaller adaptation or no adaptation at all (Fig. 6). While Systemizing abilities (SQ-R) was not a significant predictor of the differential adaptation in communicative actions, it does have a strong and overlapping relationship with NCS, and appears to be a promising predictor of differences in communicative abilities. While we only investigated female participants who tend to have higher EQs (Baron-Cohen \& Wheelwright, 2004; Focquaert, Steven, Wolford, Colden, \& Gazzaniga, 2007), we would predict even greater differences in adaptation exist when both genders are studied based on the wider range of EQ and NCS scores. This observation suggests that biased sampling of heterogeneous adult communicative abilities, usually not considered in theoretical models of communication, may lead to disparate empirical results.

This report provides novel empirical evidence on the role of audience design in human communication, while emphasizing the relevance of trait variables and joint goals in studies of communicative behavior. We distinguish effects of performance from those of prior beliefs, showing that humans approach communicative interactions by incorporating a conceptual model of the addressee and subsequently adjusting its parameters to the effectiveness of the communicative exchange, similar to anchoring-and adjustment (Tversky \& Kahneman, 1974; Epley \& Gilovich, 2006). These results indicate that nonverbal human communicative interactions (without pre-existing communicative conventions) are selected according to a socio-centric perspective and this may be a similar preliminary process to more complex communications such as speech (Clark, 1996; Isaacs \& Clark, 1987; Levinson, 2006).

\section{Acknowledgments}

Authors' contribution: experimental design (S.E.N.N., M.L.N., I.T.), data collection (S.E.N.N., M.L.N., R.D.N.N., I.A.C.V.), data analysis (S.E.N.N., M.L.N., R.D.N.N, I.T.), manuscript preparation (S.E.N.N., M.L.N., R.D.N.N., I.A.C.V., J.P.R., P.H., I.T.). This work has been completed in the context of the European Union Integrated Project JAST (Joint Action Science and Technology), Grant FP6-IST2-003747. We would like to thank Asli Ozyurek for her advice and enthusiasm. Also thanks to Valeney Saraswathy Toni and Kirsten Weber for being our gracious "child" and "adult". Dedicated to Dr. Topher Bill, who lives in the hearts and minds of his students.

\section{Appendix A. Partner questionnaire}

Below are several questions that we would like you to answer about your partners in the game. Please fill them out as best as you can and as honestly as possible.

\section{Your younger partner $^{* *}$}

How old do you think your younger partner is?

Do you think your younger partner has used a computer before?

If so, how often do they use it?

How alike do you think your younger partner is to you on scale or $1-10,10$ being exactly like you, 1 being nothing like you?

Do you think your younger partner ......**

\begin{tabular}{l|l}
\hline \multicolumn{2}{c}{ YES } \\
\hline Rides a bike & \\
Can Count to 100 & \\
Can do multiplication problems & \\
Knows the rules for driving a car & \\
Owns stuffed toys/animals & \\
Plays video games & \\
Goes to the store on their own & \\
Has read The Diary of Anne Frank & \\
Can sing the song, "Zakdoekje Leggen" &
\end{tabular}

** All questions and order of adult/child questions were counterbalanced across subjects.

Your older partner $^{* *}$

How old do you think your older partner is?

Do you think your older partner has used a computer before?

If so, how often do they use it?

How alike do you think your older partner is to you on scale or $1-10,10$ being exactly like you, 1 being nothing like you? 
Do you think your older partner ......***

\begin{tabular}{|c|c|}
\hline YES & NO \\
\hline \multicolumn{2}{|l|}{ Rides a bike } \\
\hline \multicolumn{2}{|l|}{ Can Count to 100} \\
\hline \multicolumn{2}{|l|}{ Can do multiplication problems } \\
\hline \multicolumn{2}{|l|}{ Knows the rules for driving a car } \\
\hline \multicolumn{2}{|l|}{ Owns stuffed toys/animals } \\
\hline \multicolumn{2}{|l|}{ Plays video games } \\
\hline \multicolumn{2}{|l|}{ Goes to the store on their own } \\
\hline \multicolumn{2}{|l|}{ Has read the Diary of Anne Frank } \\
\hline Can sing the song, "Zakdoekje Leggen" & \\
\hline
\end{tabular}

\section{General questions}

Did you find the game difficult? What was most difficult for you?

Do you think you behaved differently with your younger partner than with your older partner ${ }^{* *}$ ? If yes, how so?

Who do you think played better - the adult or child ${ }^{* *}$ ?

**All questions and order of adult/child questions were counterbalanced across subjects.

\section{References}

Bargh, J. A., Chen, M., \& Burrows, L. (1996). Automaticity of social behavior: Direct effects of trait construct and stereotype priming on action. Journal of Personality and Social Psychology, 71, 230-244.

Baron-Cohen, S., \& Wheelwright, S. (2004). The Empathy Quotient: An investigation of adults with Asperger syndrome or high functioning autism, and normal sex differences. Journal of Autism and Developmental Disorders, 34, 163-175.

Barr, D. J. (2004). Establishing conventional communication systems: Is common knowledge necessary? Cognitive Science, 28, 937-962.

Brand, R. J., Baldwin, D. A., \& Ashburn, L. A. (2002). Evidence from 'motionese': Modification in mothers' infant-directed action. Developmental Science, 5(1), 72-83.

Brodsky, P., Waterfall, H., Edelman, S. (2007). Characterizing Motherese: on the computational structure of child-directed language, in Proc. Cognitive Science Society Conference.

Brown-Schmidt, S., Gunlogson, C., \& Tanenhaus, M. K. (2008). Addressees distinguish shared from private information when interpreting questions during interactive conversation. Cognition, 107(3), $1123-1134$.
Cacioppo, J. T., Petty, R. E., \& Kao, C. F. (1984). The efficient assessment of need for cognition. Journal of Personality Assessment, 48, 306-307.

Cacioppo, J. T., Petty, R. E., Feinstein, J. A., Blain, W., \& Jarvis, G. (1996). Dispositional differences in cognitive motivation: The life and times of individuals varying in need for cognition. Psychological Bulletin, 2, 197-253.

Clark, H. H., \& Carlson, T. B. (1982). Hearers and speech acts. Language, 58, $332-373$

Clark, H. H. (1996). Using language. Cambridge: Cambridge University Press.

De Ruiter, J. P., Noordzij, M., Newman-Norlund, S., Hagoort, P., \& Toni, I. (2007). On the origin of intentions. In P. Haggard, Y. Rossetti, \& M. Kawato (Eds.), Attention and Performance XXII: Sensorimotor foundation of higher cognition (pp. 593-610). Oxford: Oxford University Press.

Epley, N., \& Gilovich, T. (2006). The anchoring-and-adjustment heuristic. Why the adjustments are insufficient. Psychological Science, 17(4), 311-318.

Evans, C. J., Kirby, J. R., \& Fabrigar, L. R. (2003). Approaches to learning, need for cognition, and strategic flexibility among university students. British Journal of Educational Psychology, 73, 507-528.

Focquaert, F., Steven, M. S., Wolford, G. L., Colden, A., \& Gazzaniga, M. S. (2007). Empathizing and systemizing cognitive traits in the sciences and humanities. Personality and Individual Differences, 43, 619-625.

Garfinkel, H. (1967). Studies in ethnomethodology. Prentice Hall.

Grice, P. (1969). Utterer's meaning and intention. The Philosophical Review, $78,147-177$

Grieser, D. L., \& Kuhl, P. K. (1988). Maternal speech to infants in a tonal language; Support for universal prosodic features in motherese. Developmental Psychology, 24, 14-20.

Holler, J., \& Stevens, R. (2007). The effect of common ground on how speakers use gesture and speech to represent size information. Journal of Language and Social Psychology, 26, 4-27.

Horton, W. S., \& Keysar, B. (1996). When do speakers take into account common ground? Cognition, 59, 91-117.

Horton, W. S., \& Gerrig, R. J. (2005). The impact of memory demands on audience design during language production. Cognition, 96, 127-142.

Isaacs, E. A., \& Clark, H. H. (1987). References in conversation between experts and novices. Journal of Experimental Psychology: General, 116, 26-37.

Jacobs, N., \& Garnham, A. (2007). The role of conversational hand gestures in a narrative task. Journal of Memory and Language, 56, 291-303.

Keysar, B., Barr, D. J., \& Horton, W. S. (1998). The egocentric basis of language use: Insights from a processing approach. Current Directions in Psychological Science, 7, 46-50.

Krauss, R. M., \& Fussell, S. R. (1991). Perspective-taking in Communication: Representation of other's knowledge in reference. Social Cognition, 9, 2-24.

Levinson, S. C. (2006). On the human "interactional engine". In N. J. Enfield \& S. C. Levinson (Eds.), Roots of human sociality: Culture, cognition, and interaction. Berg: Oxford.

Nadig, A., \& Sedivy, J. (2002). Evidence of perspective-taking constraints in children's on-line reference resolution. Psychological Science, 13(4), 329-336.

O'Neill, M., Bard, K. A., Linnell, M., \& Fluck, F. (2005). Maternal gesture with 20-month-old infants in two contexts. Developmental Science, $8(4), 352-359$.

Ozyurek, A. (2002). Do speakers design their cospeech gesture for their addressees? The effects of addressee location on representational gestures. Journal of Memory and Language, 46, 688-704.

Ruiter, R. A. C., Verplanken, B., De Kremer, D., \& Kok, G. (2004). Danger and fear control in response to fear appeals: The role of need for cognition. Basic and Applied Social Psychology, 26(1), 13-24.

Saxe, R., \& Baron-Cohen, S. (2006). Editorial: The neuroscience of theory of mind. Social Neuroscience, 1, 1-9.

Shintel, H., \& Keysar, B. (2007). You said it before and you'll say it again: Expectations of consistency in communication. Journal of Experimental Psychology: Learning, Memory \& Cognition, 33, 357-369.

Tversky, A., \& Kahneman, D. (1974). Judgment under uncertainty: Heuristics and biases. Science, 185, 1124-1130.

Warren-Leubecker, A., \& Bohannon, J. N. III, (1984). Intonation patterns in child-directed speech: Mother-Father differences. Child Development, $55,1379-1385$.

Wheelwright, S., Baron-Cohen, S., Goldenfeld, N., Delaney, J., Fine, D., Smith, R., et al (2006). Predicting autism spectrum quotient (AQ) from the systemizing quotient-revised (SQ-R) and empathy quotient (eq). Brain Research, 1079, 47-56. 